\title{
Magnetic Resonance Imaging Distortion and Targeting Errors from Strong Rare Earth Metal Magnetic Dental Implant Requiring Revision
}

\author{
Seong-Cheol PARK ${ }^{*}$, Chong Sik $\mathrm{LEE}^{2^{*}}$, Seok Min $\mathrm{KIM}^{2^{*}}$, Eu Jene $\mathrm{CHOl}^{2}$, Do Hee LEE${ }^{1}$, Jung Kyo LEE ${ }^{1,3}$ \\ ${ }^{1}$ Asan Medical Center, Department of Neurosurgery, Seoul, Korea \\ ${ }^{2}$ Asan Medical Center, Department of Neurology, Seoul, Korea \\ ${ }^{3}$ University of Ulsan, College of Medicine, Seoul, Korea \\ ${ }^{*}$ These authors contributed equally to this study.
}

\section{ABSTRACT}

Recently, the use of magnetic dental implants has been re-popularized with the introduction of strong rare earth metal, for example, neodymium, magnets. Unrecognized magnetic dental implants can cause critical magnetic resonance image distortions. We report a case involving surgical failure caused by a magnetic dental implant. A 62-year-old man underwent deep brain stimulation for medically insufficiently controlled Parkinson's disease. Stereotactic magnetic resonance imaging performed for the first deep brain stimulation showed that the overdenture was removed. However, a dental implant remained and contained a neodymium magnet, which was unrecognized at the time of imaging; the magnet caused localized non-linear distortions that were the largest around the dental magnets. In the magnetic field, the subthalamic area was distorted by a $4.6 \mathrm{~mm}$ right shift and counter clockwise rotation. However, distortions were visually subtle in the operation field and small for distant stereotactic markers, with approximately 1-2 $\mathrm{mm}$ distortions. The surgeon considered the distortion to be normal asymmetry or variation. Stereotactic marker distortion was calculated to be in the acceptable range in the surgical planning software. Targeting errors, approximately $5 \mathrm{~mm}$ on the right side and $2 \mathrm{~mm}$ on the left side, occurred postoperatively. Both leads were revised after the removal of dental magnets. Dental magnets may cause surgical failures and should be checked and removed before stereotactic surgery. Our findings should be considered when reviewing surgical precautions and making distortion-detection algorithm improvements.

KEYWORDS: Lead revision, Magnetic resonance imaging distortion, Magnetic dental implant

\section{INTRODUCTION}

A luminum-nickel-cobalt magnets have sometimes been used for dental implants (2). Magnetic resonance imaging (MRI) distortions caused by magnetic dental implants have been known for decades $(3,14)$. However, rare earth metal, for example, neodymium and samarium magnets, are several times more powerful and durable than conventional magnets and have recently revolutionized the whole industry (10). This type of dental implant is popular in
Japan and has also been used in Europe and Korea (7). These are used as rare earth magnetic-retained overdenture support (2). This type of overdenture is attached to a magnet, which is implanted in the mandible (2). Rare earth metal magnetic implants can cause significant MRI distortions if all detachable parts, including the keeper and magnet, are not removed.

Therefore, if a rare type of magnetic dental implant is present among numerous types of non-magnetic dental implants, it may be missed when acquiring magnetic resonance (MR) 
images. In addition, distortions caused by magnets are local and non-linear, cannot be corrected, and are subtle in the operation field distant from the magnet (Figure $1 \mathrm{~A}-\mathrm{H}$ ).

We report a case involving surgical failure caused by a magnetic dental implant. Surgical failure caused by magnetic dental implant-related distortions of MRI in deep brain stimulation (DBS) patients has not been previously reported.

\section{CASE REPORT}

\section{Clinical History}

A62-year-old male patient with an 18-year history of Parkinson's disease had been treated with medications. The patient was indicated for DBS. Preoperatively, the levodopa equivalent dose was $1960 \mathrm{mg}$ and the patient was taking levodopa, sustained release levodopa, ropinirole $12 \mathrm{mg}$, and amantadine 100 mg (21). Preoperatively, the Unified Parkinson's Disease Rating Scale part III scores for medication off and on were 56 and 28 , respectively, with $50 \%$ improvements in the levodopa challenge test.

\section{Imaging and Surgical Planning Protocols}

After the application of a Leksell frame (Elekta, Stockholm, Sweden), non-contrast 1.5-mm-thick computed tomography (CT), 1.5 T MRI, including magnetization prepared rapid gradient echo for anterior commissure-posterior commissure selections and T2 sequence for target selection, were preoperatively performed (17). Subthalamic nucleus (STN) targets and entries were marked in the SurgiPlan ${ }^{\circledR}$ version 9.0 (Elekta) software using $1.5 \mathrm{~T}$ MRI defined by stereotactic markers, 1.5 T MRI co-registered to non-enhanced CT, and 3 T MRI coregistered to non-enhanced CT. MRI distortions and co-registration errors were compared with CT images in Surgiplan ${ }^{\circledR}$. After the operation, non-contrast CT images were checked to identify electrode locations and hemorrhagic complications. Postoperatively, low specific absorption rate 1.5 T MRI was checked after the removal of the frame $(4,17)$. Electrode locations were checked against in postoperative MRI (7).

\section{Surgical Procedure}

Quadripolar DBS electrodes (3389, Medtronic, Minneapolis, MN, USA) were bilaterally used for STN. Entry points were defined at a coronal suture. Target coordinates were calibrated for better targeting within 0-1 $\mathrm{mm}$ (9). Target coordinates were set in the Leksell stereotactic frame. The final electrode position was checked by the intraoperative C-arm. Postoperatively, low specific absorption rate 1.5 T MRI was checked before the removal of the frame $(4,17)$. Electrode locations were checked against postoperative MRI (7). At the first operation on June 14, 2014, the right side was operated first and the left side was operated next in a single operation session.

\section{Image Distortions and Errors in the First DBS}

During the stereotactic MRI procedure for the first DBS, the overdenture attached by the magnet force was removed. However, a special type of implant with a magnetic disc at the top was not recognized and remained in position (Figure 2AD). In the preoperative stereotactic imaging, distortions near the magnetic implants were severe with image deformation and signal loss (Figure 1A-H). Distortions in areas closer to the dental magnets were as large as $11-22 \mathrm{~mm}$ in the lower nasal septums. In the axial image at the subthalamic area level, distortion involving a shift of approximately $4.6 \mathrm{~mm}$ (Figure 1A-H) occurred. However, image shape changes were unrecognizable. Bony structure distortions were confused with normal nasal septal deviations and asymmetry at this level (8). The image distortion in the target area may have been aggravated by stereotactic marker shifts, which may have resulted in a larger total error of $\leq 5.9 \mathrm{~mm}$ (Figure $1 \mathrm{~A}-\mathrm{H})$. This distortion caused large errors at postoperative lead locations, which were $5.4 \mathrm{~mm}$ on the right side and $2.7 \mathrm{~mm}$ on the left side from the intended targets $(1,6)$.

\section{Intraoperative Microelectrode Recordings, Stimulations, and Track Selections}

In the initial operation, we routinely inserted 3 microelectrodes initially and inserted additional microelectrodes when needed. Therefore, anterior, center, and lateral electrodes were initially inserted. Right-side microelectrode recordings were obtained at the point of $-10 \mathrm{~mm}$ from the target. Because STN multiunit activities and irregular spikes were poor in three tracks, an additional two tracks (medial and posterior) were recorded (19). Among the five tracks, STN signals were found only in the anterior and posterior microelectrodes. During anterior electrode stimulation, no symptom improvement was observed. Symptoms improved with the bradykinesia and the rigidity grade decreased from 2 to 0 at $2 \mathrm{~V}, 60 \mu \mathrm{s}$, and $130 \mathrm{~Hz}$ intraoperative posterior track stimulation. From $3 \mathrm{~V}$, facial dystonia appeared, and the stimulation voltage was not increased further. However, there were no tracks better than the posterior track, and the electrode was inserted into the posterior track. The electrode tip was located at the target when checked by intraoperative radiography with a C-arm. For the left side, STN microelectrode recording signals and symptom improvement from macrostimulations were best in the lateral track. A lead was inserted into the lateral track. Because the direction of distortion was toward the right side, the selection of the lateral track (the electrode at the furthest left) was also correct and the approximate $4-\mathrm{mm}$ deviation caused by the distortion was reduced by approximately $50 \%$.

\section{Postoperative Course after the First DBS}

No hemorrhagic complications occurred. Postoperative stimulation settings to minimize side effects were right 0 - electrode, $3.5 \mathrm{~V}, 90 \mu \mathrm{s}, 90 \mathrm{~Hz}$, and left 2+1- electrode, $90 \mu \mathrm{s}$, and $90 \mathrm{~Hz}$. Initially after the first DBS, levodopa equivalent doses were $1360 \mathrm{mg}$. At 10 months after the first operation the levodopa equivalent dose increased to $1727 \mathrm{mg}$. We speculated that the stimulation-induced swallowing difficulty and drooling are related to the right pyramidal tract stimulations from the right electrode location close to these structures. Freezing of gait was not improved by the stimulation and sometimes worsened.

\section{MRI Distortion Correction by Dental Magnet Removal}

After the failure of the first surgery, we suspected MRI distortions from a dental implant as the cause. We, then, 

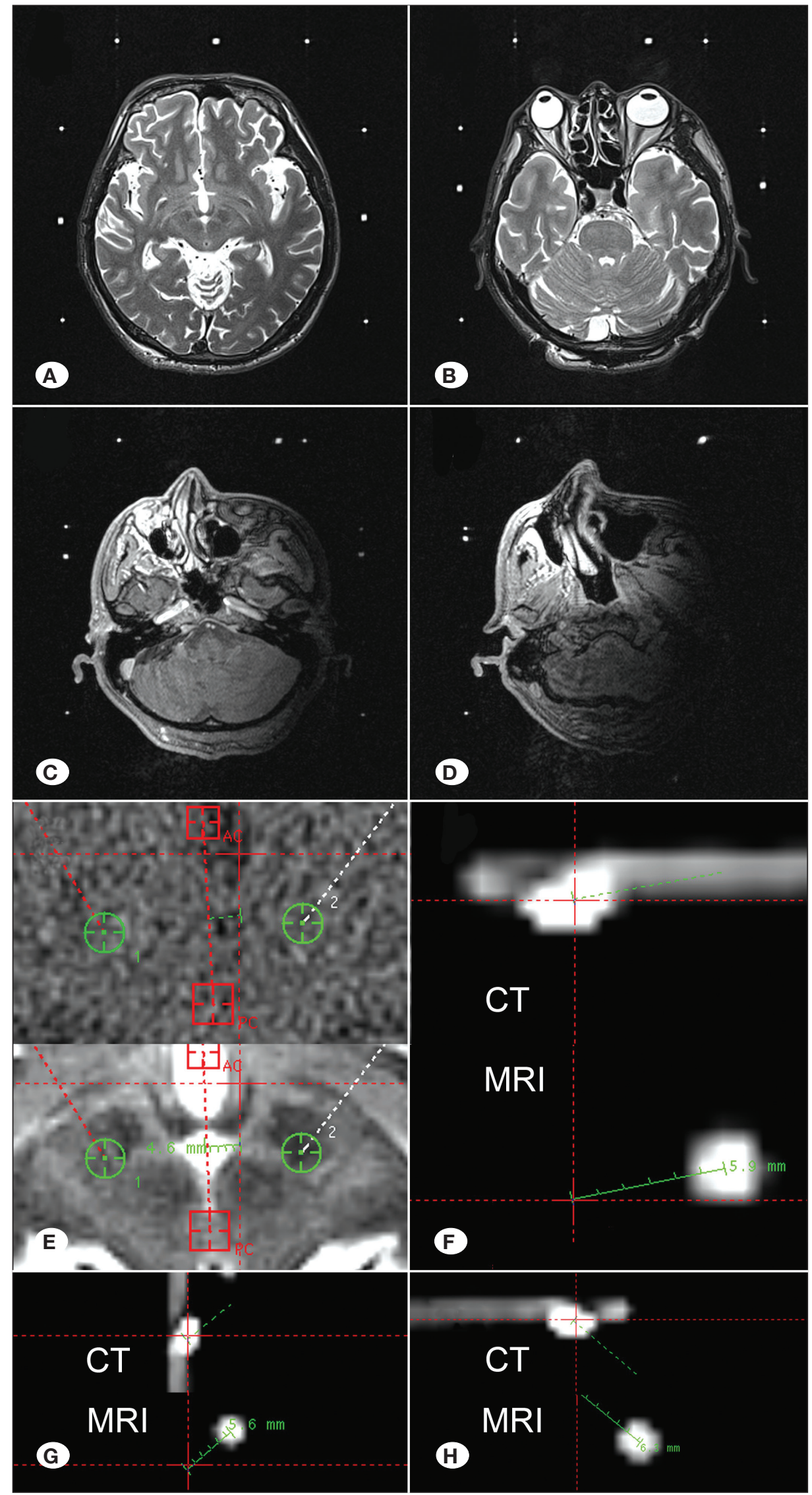

Figure 1: Subtle distortions in preoperative stereotactic MRls. A) In this axial image at the surgical target level, the distortion is subtle and difficult to recognize. However, shifts toward the right side are present in comparison to computed tomography without distortion.

B) In the lowest axial slice of the T2-weighted sequence, these distortions are still subtle and difficult to differentiate from normal anatomic variations including nasal septal deviations and facial asymmetry. C) The lower area is only covered by a magnetization prepared rapid acquisition gradient echo sequence. The distortion increases because the location is closer to dental magnetic implants.

D) The most severe distortion is shown in the lowest axial image. However, distortions were not preoperatively discovered because these images were not used for direct targeting.

E) Preoperatively, non-contrast computed tomography (upper image) and T2-weighted magnetic resonance imaging (lower image) were obtained. Soft tissue contrast in non-contrast computed tomography was rather obscure for identifying distortions and midline shifting. In CT and MR images registered by stereotactic markers, the right-side distortion was $4.6 \mathrm{~mm}$ in the subthalamic area. After this event, we preoperatively obtain contrastenhanced CTs to check distortions.

F) Anterior plate left corner stereotactic marker in CT (upper image) and MRI (lower image) co-registered based on images not using stereotactic markers. The center of red crosses is the location of the CT stereotactic marker. MRI marker locations are different by $5.9 \mathrm{~mm}$.

G) Left lateral plate anterior stereotactic marker shown by the same method. MRI markers are distorted to the anterior and left directions with a different angle.

H) Anterior plate right corner stereotactic marker shown by the same method. This marker is distorted to the left and posterior. Considering (F), (G), (H), and (E) with left deviation and clockwise rotation of stereotactic markers when images were coregistered, MRI was distorted with the shift toward the right and counter clockwise rotation. This may be related to the direction of the magnetic field. 
removed the dental magnets (Figure 2A-D). After removal, the distortions were almost completely reduced (Figure $3 A-D)$.

\section{Bilateral Lead Revision and Microelectrode Recording Tracks}

Three tracks, including the anterior, center, and lateral tracks, were used for bilateral microelectrode recordings and macrostimulations. The STN signals were better than those at the first operations. For both sides, the anterior tracks were selected on the basis of intraoperative microelectrode recordings and symptom improvement caused by stimulation (Figure 4A, B). Both leads were revised (Figure 4A, B). Postoperatively, with stimulation, the tremor grade was 0 , the bradykinesia and rigidity grades improved from 2 to 0 , gait disturbance was grade 1 , and stooped posture was grade 2. After the revision, swallowing difficulty and drooling side effects disappeared, and the patient's global impression scale of improvement for the revision was $1+(12)$. Medication was reduced to $600 \mathrm{mg}$ levodopa equivalent dose.

Three months after the revision, the stimulation frequencies were lowered from $130 \mathrm{~Hz}$ to $80 \mathrm{~Hz}$, which resulted in partial improvement in freezing of gait (20) in the lower frequency stimulation suggested in the literature (15). The patient was followed for 15 months after the revision. The medication was increased to $900 \mathrm{mg}$ per day in the last follow-up.

\section{DISCUSSION}

Structure of Magnetic Dental Implants and Stereotactic Surgical Pitfalls

Strong rare earth metal magnets in dental implants are used to support magnetic-retained overdenture (2). The magnets of these dental implants are detachable along with the keeper
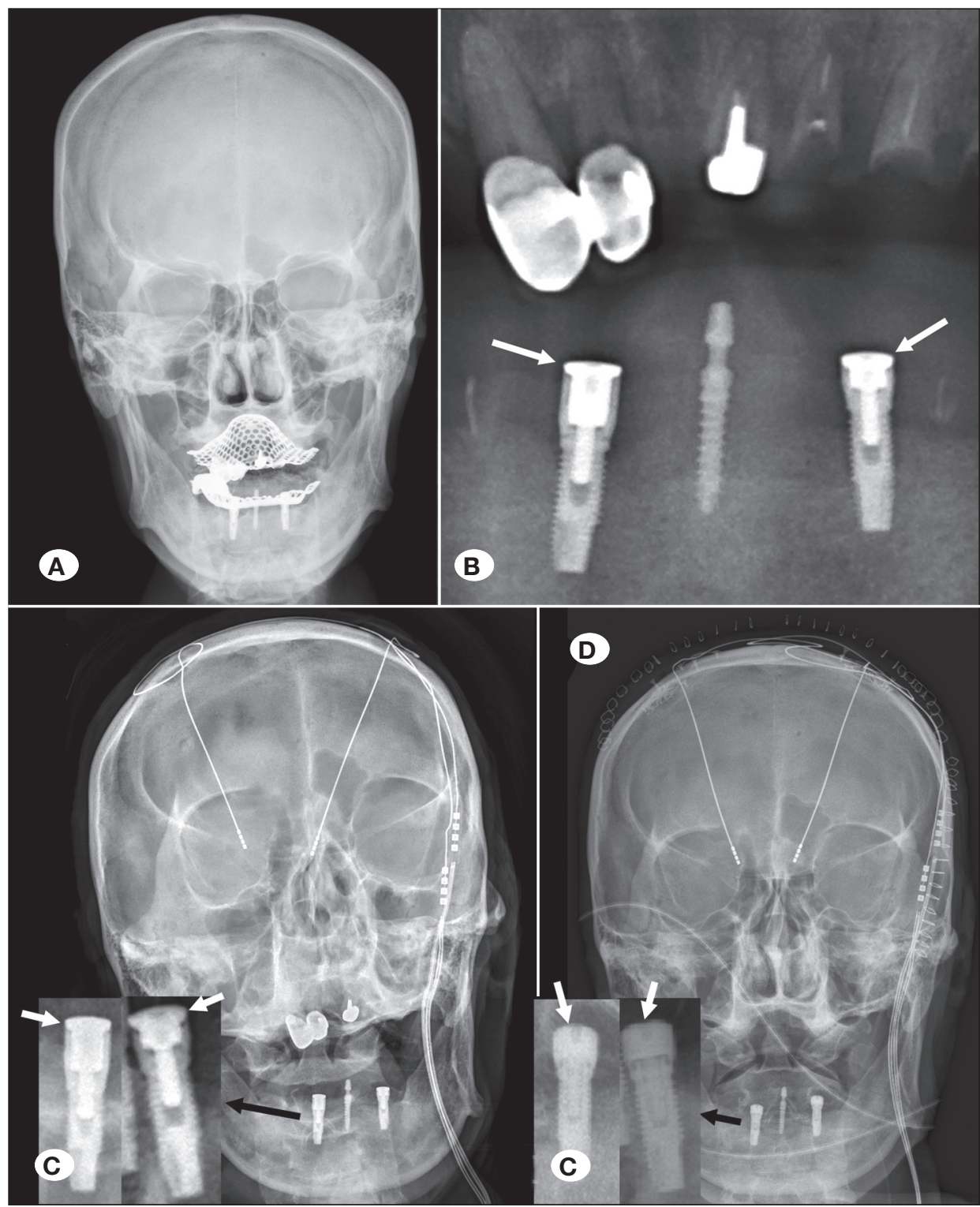

Figure 2: Skull radiographs showing dental implants.

A) Magnetic dental implants with magnetic overdenture (mesh-like structure) in place in preoperative skull radiographs.

B) Enlarged skull radiographs with magnets (white arrows) attached to the keeper and permanent implants. C) Skull radiograph after the first DBS with severe MRI distortions. Magnetic dental implants are enlarged and shown in the left lower corner (black arrow). Magnets are discs (white arrows) over the titanium implants. Because of the distortion, the right electrode was greatly misplaced. D) Skull radiograph after the second DBS without distortion and revised leads. The magnetic discs over keepers (white arrows) were removed reducing most distortions. Nonmagnetic metal implants on the maxilla were also replaced by resins to minimize metal artifacts. 
screw below it connected to the permanent implant (Figure 2A-D). To reduce MRI distortions when needed, magnetic parts are removed and replaced with non-magnetic metal parts in permanent implants (13). MRI technicians routinely remove overdentures before performing MRI. However, the removal of overdentures before $\mathrm{MRI}$ is an insufficient measure for this special type of magnetic implants. The magnet and keeper should also be removed to minimize magnetic and metal artifacts.
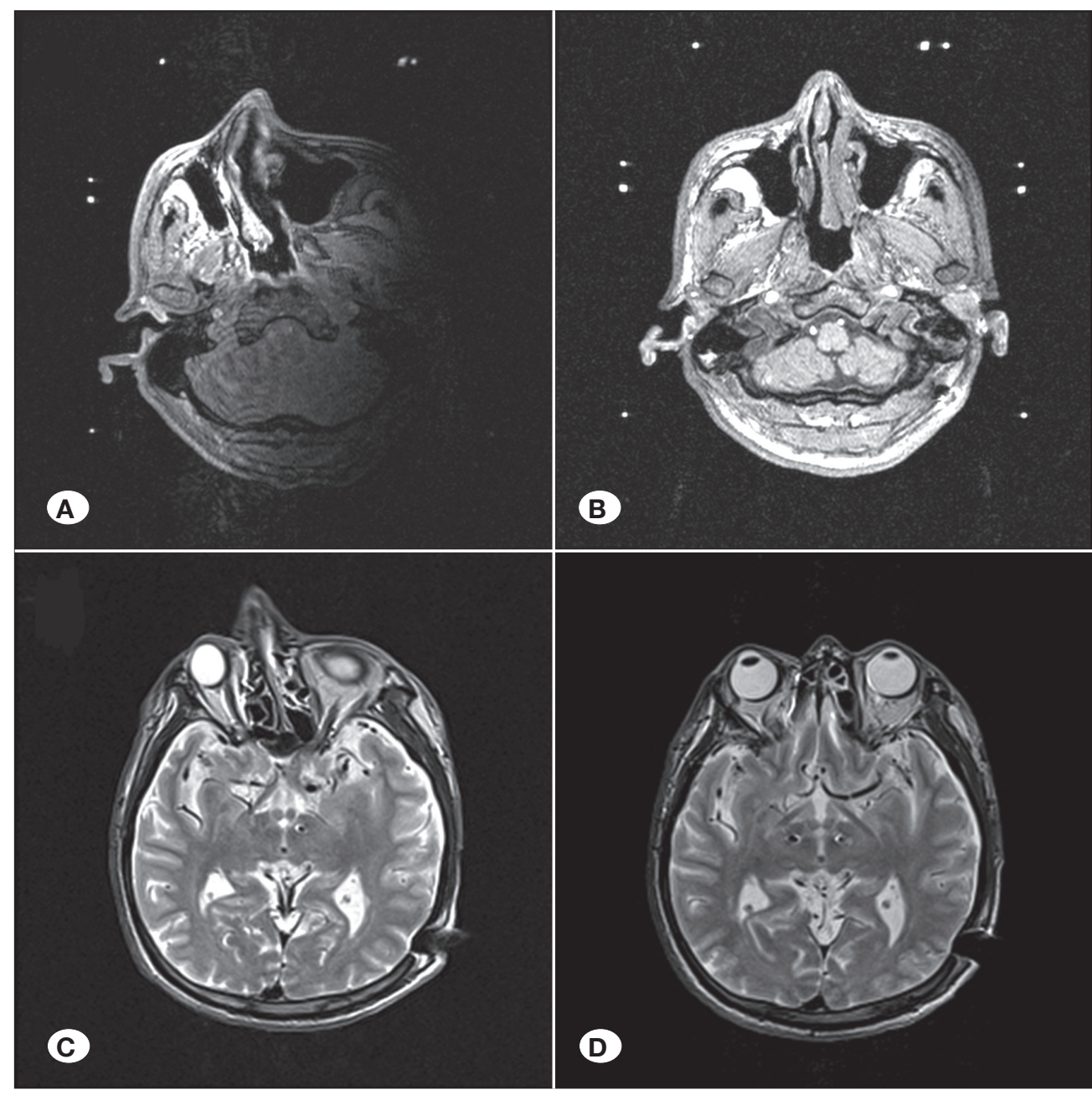

C
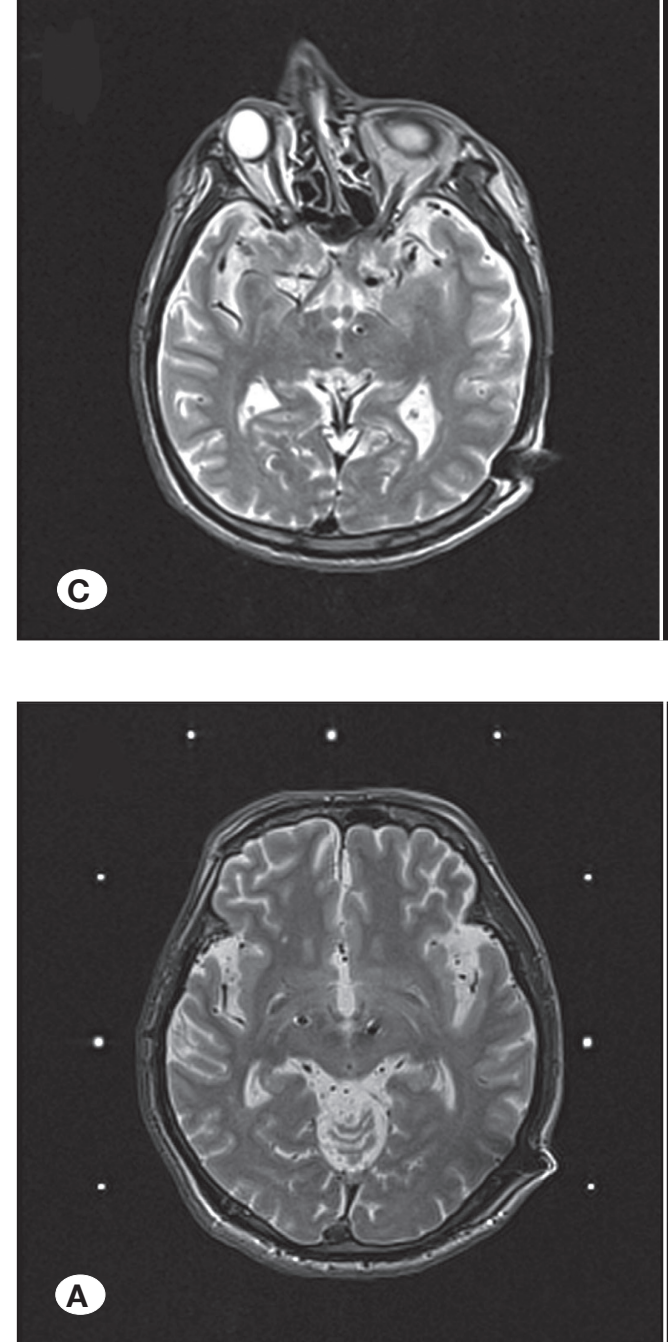

Figure 3: Severity of MRI distortions according in the presence of magnetic implants. A) In lower axial images near the upper teeth, the distortion was most severe.

B) After the removal of dental magnets and implants, the distortion was corrected.

C) In the more superiorly located axial image, the distortion was less severe because the distance from the magnet causing nonlinear local distortion increased. D) After the removal of dental neodymium magnets, the distortions were corrected.
Figure 4: Lead locations before and after revisions.

A) Preoperative stereotactic magnetic resonance imaging of the lead revision surgery.

B) After lead revisions, both B leads were moved closer to the theoretical ideal targets. 


\section{Intraoperative Correction by Microelectrode Recordings and Stimulations}

Microelectrode recordings and intraoperative stimulation showed that the selected posterior and lateral tracks were the closest to subthalamic nucleus among the five tracks. The intraoperative corrections appeared to be effective (16). However, 2-mm shifts were insufficient, particularly for the right side, to correct large errors.

\section{Criteria for Lead Revision}

Deviations of $>2 \mathrm{~mm}$ have been suggested in the literature to require revision (18). Both electrodes, particularly the right lead, clearly exceeded the acceptable range of error. Considering the ineffectiveness and side effects, the lead revision was inevitable.

\section{Non-linear Distortion Characteristics from Rare Earth Metal Magnetic Dental Implant}

Unlike typical MRI distortions, which are the greatest at the periphery and smallest at the center, the distortions were greater at the central area closest to the dental magnets in this case $(11,22)$. The characteristics of MRI distortion caused by strong rare earth metal magnets are not well known and appear to shift and rotate whole images in addition to causing great local distortions (Figures $1 \mathrm{~A}-\mathrm{H}, 3 \mathrm{~A}-\mathrm{D}$ ). In addition, the stereotactic error-checking algorithm of the software we used could not reject complex non-linear distortion errors using stereotactic marker deviations. We speculate that stereotactic markers are distant from the magnets, which results in subtle distortion and shifts and rotations of all markers together. This type of distortion was accepted in the software. When images are registered with stereotactic markers, CT and MRI stereotactic markers could be overlapped because of small, <1-2 mm errors. However, the distortion of images closer to dental magnets was much greater with $4.6 \mathrm{~mm}$ right shifts when registered using stereotactic markers. Counter clockwise rotations over $6 \mathrm{~mm}$ distances were suggested on the basis of marker displacements when we co-registered the CT and MR images on the basis of images not using stereotactic markers (Figure 1A-H). Our case shows that even large distortions can be missed when they are unevenly and subtly distributed. A different error detection algorithm may be required to automatically detect local non-linear distortions or correct distortions. It has also been suggested that non-linear $\mathrm{MRI}$ distortion cannot be fully compensated by the linear coregistration algorithm used in most software (23). Therefore, software corrections would be very difficult, so causes of distortions should be removed.

\section{Use of Contrast-enhanced CT for MRI Distortion Checks}

In a co-registration study, contrast-enhanced vessels were used as landmarks for CT-MRI fusion (5). Contrast-enhanced CT better visualizes vessels, and these can be directly compared with vessels on MR images to check distortions when images are registered using stereotactic markers (5). Non-contrast CT could not easily and conspicuously identify a deviated midline of the soft tissue (Figure 1A-H).

\section{Nasal Septal Deviation as an Indicator of Large MR Distortions}

A crooked nose can be a sign of MRI distortion (Figure 3A-D). However, these MRI axial slices are lower than neurosurgical targets and may be unrecognized during surgical planning. In addition, a deviated nasal septum is a common normal variation (8) and cannot be used as a definite indicator of a subtle MRI distortion.

\section{Routine Precautions to Detect MRI Distortions}

Obtaining a preoperative dental history and questioning the patient specifically about magnetic implants are important tasks. The specific shape of magnetic dental implants (Figure 2A-D) can be identified in skull radiographs. All axial images distant from surgical targets should be inspected for unusual distortions. Contrast-enhanced CT can be used for comparison to accurately check distortions.

\section{CONCLUSION}

Knowledge on magnetic dental implants and taking appropriate precautions are important in stereotactic surgeries. Non-linear distortions in the operative field and in stereotactic markers may be subtle and can be missed by both surgeons and the software. This case illustrates the surgical precautions that should be considered or taken, and the need for distortion detection or correction software algorithms.

\section{ACKNOWLEDGEMENT}

This work was supported by the Asan Medical Center [grant number 2016-7024] (50\%).

This research was supported by Basic Science Research Program through the National Research Foundation of Korea (NRF) funded by the Ministry of Education (2016R1C1B1012374) (50\%).

\section{- REFERENCES}

1. Bejjani BP, Dormont D, Pidoux B, Yelnik J, Damier P, Arnulf I, Bonnet AM, Marsault C, Agid Y, Philippon J, Cornu P: Bilateral subthalamic stimulation for Parkinson's disease by using three-dimensional stereotactic magnetic resonance imaging and electrophysiological guidance. J Neurosurg 92:615-625, 2000

2. Ceruti P, Bryant SR, Lee JH, MacEntee MI: Magnet-retained implant-supported overdentures: Review and 1-year clinical report. J Can Dent Assoc 76:a52, 2010

3. Costa AL, Appenzeller S, Yasuda CL, Pereira FR, Zanardi VA, Cendes F: Artifacts in brain magnetic resonance imaging due to metallic dental objects. Med Oral Patol Oral Cir Bucal 14:E278-282, 2009

4. Derrey S, Maltete D, Chastan N, Debono B, Proust F, Gerardin E, Weber J, Mihout B, Freger P: Deep brain stimulation of the subthalamic nucleus in Parkinson's disease: Usefulness of intraoperative radiological guidance. The Stereoplan. Stereotact Funct Neurosurg 86:351-358, 2008 
5. Fiegele T, Feuchtner G, Sohm F, Bauer R, Anton JV, Gotwald T, Twerdy K, Eisner W: Accuracy of stereotactic electrode placement in deep brain stimulation by intraoperative computed tomography. Parkinsonism Relat Disord 14:595599, 2008

6. Foltynie T, Zrinzo L, Martinez-Torres I, Tripoliti E, Petersen E, Holl E, Aviles-Olmos I, Jahanshahi M, Hariz M, Limousin $P$ : MRI-guided STN DBS in Parkinson's disease without microelectrode recording: Efficacy and safety. J Neurol Neurosurg Psychiatry 82:358-363, 2011

7. Gonda T,Maeda Y: Why are magnetic attachments popular in Japan and other Asian countries? Japanese Dental Science Review 47:124-130, 2011

8. Hajeer MY, Ayoub AF, Millett DT: Three-dimensional assessment of facial soft-tissue asymmetry before and after orthognathic surgery. Br J Oral Maxillofac Surg 42:396-404, 2004

9. Holl EM, Petersen EA, Foltynie T, Martinez-Torres I, Limousin $\mathrm{P}$, Hariz MI, Zrinzo L: Improving targeting in image-guided frame-based deep brain stimulation. Neurosurgery 67:437447, 2010

10. Jones N: Materials science: The pull of stronger magnets. Nature 472:22-23, 2011

11. Kondziolka D, Dempsey PK, Lunsford LD, Kestle JR, Dolan EJ, Kanal E, Tasker RR: A comparison between magnetic resonance imaging and computed tomography for stereotactic coordinate determination. Neurosurgery 30:402406; discussion 406-407, 1992

12. Martinez-Martin P, Rodriguez-Blazquez C, Forjaz MJ, de Pedro J, Spanish-American Longitudinal PDPSG: The clinical impression of severity index for Parkinson's disease: International validation study. Mov Disord 24:211-217, 2009

13. Masumi S, Nagatomi K, Miyake S, Toyoda S: Removable magnetic dental attachment that permits magnetic resonance imaging. J Prosthet Dent 68:698-701, 1992

14. Mathew CA, Maller S, Maheshwaran: Interactions between magnetic resonance imaging and dental material. J Pharm Bioallied Sci 5:S113-116, 2013
15. Moreau C, Defebvre L, Destee A, Bleuse S, Clement F, Blatt JL, Krystkowiak P, Devos D: STN-DBS frequency effects on freezing of gait in advanced Parkinson disease. Neurology 71:80-84, 2008

16. Pollak P, Krack P, Fraix V, Mendes A, Moro E, Chabardes S, Benabid AL: Intraoperative micro- and macrostimulation of the subthalamic nucleus in Parkinson's disease. Mov Disord 17 Suppl 3:S155-161, 2002

17. Pollo C, Vingerhoets F, Pralong E, Ghika J, Maeder P, Meuli $\mathrm{R}$, Thiran JP, Villemure JG: Localization of electrodes in the subthalamic nucleus on magnetic resonance imaging. J Neurosurg 106:36-44, 2007

18. Richardson RM, Ostrem JL, Starr PA: Surgical repositioning of misplaced subthalamic electrodes in Parkinson's disease: Location of effective and ineffective leads. Stereotact Funct Neurosurg 87:297-303, 2009

19. Schlaier JR, Habermeyer C, Warnat J, Lange M, Janzen A, Hochreiter A, Proescholdt M, Brawanski A, Fellner C: Discrepancies between the MRI- and the electrophysiologically defined subthalamic nucleus. Acta Neurochir (Wien) 153:23072318, 2011

20. St George RJ,Nutt JG, Burchiel KJ, Horak FB: A metaregression of the long-term effects of deep brain stimulation on balance and gait in PD. Neurology 75:1292-1299, 2010

21. Tomlinson CL, Stowe R, Patel S, Rick C, Gray R, Clarke CE: Systematic review of levodopa dose equivalency reporting in Parkinson's disease. Mov Disord 25:2649-2653, 2010

22. Yu C, Apuzzo ML, Zee CS, Petrovich Z: A phantom study of the geometric accuracy of computed tomographic and magnetic resonance imaging stereotactic localization with the Leksell stereotactic system. Neurosurgery 48:1092-1098; discussion 1098-1099, 2001

23. Zrinzo L, Hariz M: Errors of image coregistration may necessitate intraoperative refinement in functional neurosurgery. $J$ Neurol Surg A Cent Eur Neurosurg 74:335-336, 2013 